CAHIERS DE

NARRATOLOGIE

\section{Cahiers de Narratologie}

Analyse et théorie narratives

32 | 2017

Récit et argumentation, interactions, lieux et dispositifs sociaux

\title{
Promesses et limites de la démocratie électronique : le cas des « 5 Etoiles » en Italie
}

\section{Valentina Tirloni}

\section{(2) OpenEdition}

Journals

Electronic version

URL: http://journals.openedition.org/narratologie/7792

DOI: $10.4000 /$ narratologie. 7792

ISSN: 1765-307X

Publisher

LIRCES

Electronic reference

Valentina Tirloni, «Promesses et limites de la démocratie électronique : le cas des « 5 Etoiles » en Italie», Cahiers de Narratologie [Online], 32 | 2017, Online since 21 December 2017, connection on 01 May 2019. URL : http://journals.openedition.org/narratologie/7792 ; DOI : 10.4000/narratologie.7792

This text was automatically generated on 1 May 2019.

Article L.111-1 du Code de la propriété intellectuelle. 


\title{
Promesses et limites de la démocratie électronique : le cas des « 5 Etoiles » en Italie
}

\author{
Valentina Tirloni
}

1 Depuis que les nouvelles technologies se sont mises au service de la communication politique, la politique s'est vue transformée. D'une part, les nouvelles technologies ont permis une diffusion plus fine du message politique des partis, en espérant rejoindre le plus grand nombre d'électeurs, en produisant une forme de discours politique très redevable au storytelling américain. D'autre part, les nouvelles technologies ont aussi remis en cause certaines dynamiques de l'agir collectif politique, en faisant émerger de nouveaux acteurs politiques, de nouvelles formes d'engagement politique, ainsi qu'une remise en cause du mécanisme démocratique lui-même. Pour illustrer ce dernier point, nous souhaitons prendre comme exemple le cas du Mouvement politique « 5 Etoiles », qui a, depuis presqu'une décennie, changé les règles du jeu politique en Italie. Après une reconstitution historique des principales étapes qui ont marqué l'ascension de ce mouvement, nous voulons en analyser le type de storytelling exploité par l'inspirateur et fondateur du mouvement, Beppe Grillo. Dans la dernière partie, nous analyserons si, à partir du storytelling fondateur, le mouvement 5 Etoiles a su rester fidèle à ses principes.

\section{La communication politique face aux nouvelles technologies de communication}

2 La communication des partis politiques construit, par le biais d'un langage ${ }^{1}$ bien sciemment choisi, un discours politique, en s'inspirant des faits, tout en leur donnant une interprétation subjective qui s'adapte mieux à l'idéologie du parti. Produire une représentation du monde pour trouver une solution aux problèmes socio-économiques qui l'accablent est déjà le mettre en forme - l'in-former -à travers les catégories de perception du monde propre au parti. Celles-ci oscillent entre empirisme et nominalisme dont l'antagonisme reste inconciliable, comme bien l'illustre la fiction dystopique 1984 
d'Orwell ${ }^{2}$ où deux visions du monde, parfaitement crédibles, s'affrontent: selon la perspective empiriste les mots reflètent le réel comme un miroir le monde, alors que pour les nominalistes, défenseurs d'un déterminisme linguistique, la réalité n'existe que dans les consciences des individus par le biais du langage, avec le corollaire dangereux que celui qui contrôle le langage, contrôlera aussi ces consciences, en façonnant le monde selon les besoins de sa propagande politique. Il serait naîf de penser autrement, malgré les sympathies ou les convictions politiques qui nous animent et qui déterminent aussi l'efficacité de notre engagement politique. Nous sommes, en quelque sorte, séduits par la rationalité d'une idéologie, séduits par la justesse des fondements de sa pensée politique, convaincus par la possibilité de sa mise en place pratique, attirés par le charisme politique d'un chef de parti, déçus par l'inanité de la classe politique, bouleversés par l'énième scandale politique plus ou moins étouffé, nous vibrons de plaisir lorsque la politique renforce nos espoirs de réussite, rassure nos craintes, satisfait nos attentes. La politique dans nos sociétés démocratiques est plus que jamais dominée par les émotions et les passions, par un pathos, qui éveille des émotions triviales et simples mais bien ancrées dans les profondeurs de notre être humain. L'ancrage dans les passions alimente en nous un imaginaire politique susceptible de nous pousser à participer à la politique et même à agir. Si une des fonctions principales de la communication est celui de convaincre, de persuader, cela est encore plus vrai pour la communication politique dont on peut mesurer quantitativement le succès sur la base du nombre de voix exprimées. Convaincre donc est in primis le but d'une bonne communication politique.

3 Depuis quelques temps, cette dernière s'est beaucoup alimentée de la théorie du storytelling, de matrice nord-américaine : au-delà des points du programme politique, qui semblent avoir perdu leur efficacité persuasive, il faut surtout savoir raconter une histoire légitimante du pouvoir, un récit capable de susciter l'adhésion non plus sur la base d'éléments argumentatifs rationnels, mais sur la base d'une cohérence narrative fondatrice.

4 Selon Christian Salmon le storytelling est une technique de communication, de pouvoir et de contrôle qui peut aussi se révéler une puissante arme de désinformation : on produit une histoire (calquée sur des protocoles d'expérimentation psychologique sur des cobayes) qui impose à l'auditeur citoyen une "expérience tracée ${ }^{3}$, une conduite manipulée et pré-organisée. Le caractère formatant du storytelling est dû au fait qu'il "plaque sur la réalité des récits artificiels, bloque les échanges, sature l'espace symbolique de séries et de stories. Il ne raconte pas l'expérience passée, il trace les conduites et oriente les flux d'émotions $»^{4}$. Celui qui écoute l'histoire est comme par enchantement entrainé dans la trame narrative et se fait transporter là où le chanteur ambulant d'histoire l'amène, ni plus ni moins comme dans un conte de fées. Comme le remarque l'auteur à propos du marketing narratif, le but n'est pas seulement de convaincre le consommateur à acheter, mais « de le plonger dans un univers narratif, de l'engager dans une histoire crédible. Il ne s'agit plus de séduire ou de convaincre, mais de produire un effet de croyance $»^{5}$. L'histoire devient crédible si le narrateur sait bien la raconter. D'où l'importance pour les hommes et femmes politiques 2.0 de savoir se servir des nouvelles technologies pour mieux diffuser leurs histoires pré-confectionnées.

5 Le recours à l'émotion des discours politiques modernes, a, plus qu'avant la déferlante des nouvelles technologies, changé le politique: en lieu et place du débat avec échange mutuel d'arguments rationnels sur telle ou telle question politique, on assiste à la mise en récit d'histoire émouvantes, crédibles, possibles, envisageables, faisant appel surtout à 
l'adhésion sensible des électeurs. Au logos, on a subrepticement substitué le pathos. Une des passions dont on a parlé le plus souvent est l'indignation, qui, comme le remarque Pierre Le Coz, est « l'émotion révolutionnaire par excellence " 6 , car elle «tire son énergie affective de notre nature ${ }^{7}$, en comprenant un versant primaire et viscéral comme la colère. Sur cette émotion, le Mouvement 5 Etoiles a en effet pu bâtir un storytelling très puissant, en se proposant, par la voix de Beppe Grillo, qui est souvent représenté en colère dans les médias italiens, comme le peuple des indignés, de ceux qui ne tolèrent plus les scandales politiques à répétition, de ceux qui veulent réformer la politique pour faire en sorte que les citoyens s'approprient le politique et participent au processus démocratique directement sans représentation.

\section{Le cas du Mouvement 5 Etoiles (M5E)}

6 Comment se fait-il qu'en Italie on a pu croire à la mise en forme du monde politique italien racontée par Beppe Grillo, fondateur du mouvement? Quelles conditions ont-elles permis à certains citoyens de voir une alternative politique crédible et envisageable par rapport au système traditionnel des partis ? Plusieurs facteurs ont sans doute contribué à ce succès politique dont il faudra attendre les prochaines élections parlementaires pour savoir s'il sera confirmé : d'une part, le rôle des médias traditionnels dans l'échiquier politique, d'autre part le mécontentement diffus des citoyens italiens, de plus en plus désorientés face aux comportements des hommes et femmes politiques.

7 Pour mieux comprendre l'émergence de ce phénomène socio-politique, nous allons en parcourir les étapes ${ }^{8}$, sous forme chronologique.

8 Tout d'abord, en Italie, comme d'ailleurs en bien d'autres pays démocratiques, il existe une forte compénétration entre la sphère du politique et la sphère des médias. Pour reprendre le modèle "médiatique» de la communication politique, développé par Gianpietro Mazzoleni ${ }^{9}$, l'interaction entre les acteurs politiques, les citoyens et les médias advient à l'intérieur d'un espace public médiatisé et médiatique : les médias, autrement dit, s'identifient à l'espace public et sont à la fois les canaux des échanges entre la classe politique et les citoyens, et aussi des interlocuteurs capables de conditionner les rapports entre acteurs politiques, en influençant la nature de ces relations et en obligeant les institutions, les politiciens, les citoyens à adopter les logiques de communication de masse. La montée en puissance du phénomène Silvio Berlusconi en a été une très explicite illustration: la télévision a été le principal canal de diffusion de la communication politique, selon un effet de spectacularisation et de personnalisation du politique.

9 Sur ce terrain bien semé, où la communication politique rend visible seulement ceux qui ont accès à la diffusion des messages politiques, restaient exclus et les personae non gratae au système politique traditionnel, et ceux qui ne détiennent pas les moyens de communication. L'arrivée de l'Internet a sans doute modifié cet état de chose, cristallisé autour d'un système auto-conservateur et tendant à se reproduire lui-même. La possibilité donc de diffuser par d'autres voies médiatiques un message politique, ou d'une autre nature, a trouvé en Internet un puissant moyen de réalisation. Il est maintenant possible de ne pas attendre le licet des médias traditionnels pour faire de la communication politique. De façon analogue, en France, on remarquera comment JeanLuc Mélenchon a pu court-circuiter les médias traditionnels en publiant directement sur le réseau social YouTube ses propres vidéos lors de la dernière campagne présidentielle de 2017. Avec Internet, les médias traditionnels se trouvent en compétition avec un nouvel 
acteur/moyen de communication capable de remettre en cause la véritable construction ou information de la réalité. De plus, la prolifération incontrôlée de toutes sortes d'informations (du médical au politique, en passant par les cultures alternatives, les modes et styles de vie) a en effet remis en cause la sorte de monopole informationnel des médias traditionnels. Une autre source d'information, plus collaborative et participative, est à disposition de tout le monde. Encore faut-il savoir faire le tri parmi les sources incontrôlées ou anarchiquement constituées d'Internet. Sommes-nous en mesure de faire la part des choses lorsqu'on trouve une information sur Internet? Avons-nous une quelconque garantie de la fiabilité ou du fondement d'une nouvelle ainsi répandue ? Quel storytelling est-il le plus en mesure de nous assurer la vérité des faits?

10 Dans ce contexte, Beppe Grillo propose une alternative à la communication politique italienne, en réussissant à fédérer un public certes déçu mais pas encore résigné. Pour mieux comprendre, il faut, tout d'abord, reconstituer l'histoire, en parcourant brièvement sa carrière. Ancien comique de spectacles télévisés et théâtraux, Beppe Grillo concentre sa veine artistique sur la satire politique des partis et de la corruption des mœurs des politiciens italiens. A partir des années 90, il abandonne la télévision pour se consacrer davantage aux spectacles à ciel ouvert, en instituant un tout autre rapport avec son public. En particulier, il s'intéresse aux questions environnementales et met, avec succès, son charisme artistique au service d'une série de campagnes sur les biotechnologies, l'information, les droits des citoyens, la protection environnementale et contre le capitalisme ou le système bancaire mondial. En 2005, Beppe Grillo ouvre son blog personnel (beppegrillo.it, accessible aussi en anglais) qui sera consacré en 2009 au septième classement mondial par la revue Forbes ${ }^{10}$. Ce blog se veut un lieu de discussion et d'information et se transforme vite en plateforme pour le lancement d'actions collectives. Parmi les campagnes d'action proposées, on citera la requête de retirer l'armée italienne de l'Iraq, l'action « Parlement propre » pour éloigner de cette assemblée les députés condamnés définitivement par la Cour de Cassation, l'action pour la protection du travail précaire et bien d'autres actions capables de catalyser un grand nombre de followers ante litteram, là où la presse nationale et les médias étaient restés silencieux.

11 Grâce au succès inespéré obtenu avec le blog, Beppe Grillo envisage qu'une véritable action politique de protestation puisse prendre forme, en invitant les citoyens intéressés à communiquer et à échanger entre eux, voire à se rencontrer sur leurs propres territoires. Il décide ainsi de créer les Meetup, des groupes de rencontres à base territoriale : l'on rappellera le premier, organisé à Turin contre la ligne TGV en Valle de Susa le 17 décembre 2005 et celui organisé à Naples contre la (mauvaise) gestion des déchets ${ }^{11}$. Ces initiatives de mobilisation montrent bien à B. Grillo et à ses collaborateurs le potentiel d'Internet et son pouvoir viral fédérateur qui semble récupérer l'énergie de l'ancien activisme socio-politique que les partis politiques traditionnels semblent avoir abandonné, laissant la place à l'omniprésence de la télévision.

12 A ce stade de l'histoire du mouvement, le phénomène commence à se transformer du virtuel au réel, notamment avec l'organisation du V-Day, où le $\mathrm{V}$ a une triple signification: le signe de victoire de Churchill, le V de Vendetta, la bande dessinée de Alan Moore et David Llyod et le bien plus prosaïque «va te faire... $»^{12}$ ou selon une autre interprétation attribuée à Beppe Grillo lui-même, le V-Day se situe entre le D-Day du débarquement en Normandie des troupes alliées, et le V de Vendetta, pour souligner que, depuis 1943 , rien n'a changé dans la politique italienne, rien n'a été résolu ${ }^{13}$. Le V-Day, qui 
n'est pas sans rappeler le rituel orwellien des deux minutes de haine, se divise en deux parties : une consacrée à la protestation contre la caste politique italienne au cri explicite de "vaff... ", l'autre à la récolte de signatures pour la présentation de lois d'initiative populaire, en accord avec la Constitution italienne qui octroie ce droit directement aux citoyens et pas seulement au Parlement.

$13 \mathrm{Au}$ vu de la montée croissante des Meetups et des V-Day, Grillo exhorte ses followers à présenter des listes citoyennes aux élections territoriales de 2008, en se portant garant des listes civiques proposées, garant du respect des principes affirmés dans le storytelling des Grillini : transparence, casier judiciaire vierge, se faire porte-parole du peuple, être choisi par les internautes inscrits au mouvement. Les principes du mouvement racontés par le storytelling grillino et auxquels tout adhérent ou potentiel candidat doit obéir sont : critique du mauvais fonctionnement de la politique, appel contre la corruption morale, sauvetage de la crise démocratique des partis, des journalistes, de l'Administration ${ }^{14}$.

C'est donc en 2009 que Beppe Grillo fonde son mouvement en s'inspirant de l'Association des Communes vertueuses qui attribue une étoile pour chacun des services suivants: l'énergie, la connectivité, l'eau, le tri des déchets, les services sociaux ${ }^{15}$. Dès la fondation aux élections administratives de 2012 et politiques de 2013, le mouvement obtient des succès évidents, en s'attribuant, lors des dernières, le même pourcentage que les partis traditionnels. Mais avec le succès, il est impératif de savoir organiser une structure institutionnelle capable d'agir non plus seulement à un niveau local, mais national.

C'est avec ce changement d'échelle que le Mouvement 5 Etoiles commence à montrer les premiers signes de contradiction avec le storytelling fondateur. En effet, lorsque B. Grillo s'associe à l'entreprise de communication de Roberto Casaleggio (mort en 2016) et de son fils Davide, qui en a pris le relais, les premières tensions entre la gouvernance du mouvement et les followers commencent à éclater, en particulier avec des accusations axées sur le caractère peu démocratique du mouvement, l'autoritarisme de Grillo, le manque total de transparence sur les procédures de sélection des candidats aux différentes élections. Un exemple en est le refus de Grillo d'appuyer la candidate à la Mairie de Gênes, bien que élue par la majorité des internautes inscrits au mouvement, mais considérée comme une opportuniste non fidèle aux principes des 5 Etoiles qui souhaiterait monter sur le char des gagnants. Ces accusations vont en effet mettre en discussion le modus operandi des 5 Etoiles qui, semble-t-il, finit par reproduire les mêmes schémas de comportement, réputés obscurs, des partis traditionnels ${ }^{16}$. De plus, le storytelling initial semble marqué par un manque de culture institutionnelle et par une éthique enfantine qui, si elle fonctionne en période de crise politique, n'arrive pas en revanche à se traduire en action sur la longue durée ${ }^{17}$, en montrant ainsi le vide programmatique.

16 Et pourtant, dans le storytelling et les actions menées par les 5 Etoiles, ne manquaient pas les bonnes intentions. Il y avait l'idée de redonner la voix (et pas seulement les voies au moment des élections) aux citoyens, en expérimentant une forme de démocratie participative qui restitue la souveraineté aux citoyens et pas à un seul leader charismatique et en les impliquant activement et de façon continue dans le processus délibératif que la Constitution de la République octroie au Parlement. Une solution s'ébauche dans l'idée de démocratie " continue », proposée par Rodotà : « des mécanismes très différents de ceux de type représentatif, notamment parce que les citoyens peuvent les adopter sans recourir à des médiations ; de même ces mécanismes ne s'identifient pas à ceux de la démocratie directe, généralement associés à l'instant de la décision finale ou 
à la présence d'un processus particulier de décision ${ }^{18}$ ». Le mouvement développait aussi une critique farouche contre les médias, accusés d'une spectacularisation vulgaire et partiale, d'une mise en forme unilatérale de la réalité. Il y avait aussi la bonne intention que le citoyen aurait pu devenir acteur principal de la vie politique et que chacun aurait pu contribuer au bien et à l'intérêt général sur la base de ses compétences. Il y avait surtout l'idée d'une hétérarchie ${ }^{19}$, c'est-à-dire un réseau qui n'est pas fondé sur la hiérarchie (comme dans les partis traditionnels), mais sur des compétences qui circulent librement selon les besoins contingents, en collaborant tous ensemble, en alimentant ainsi l'intelligence collective.

17 On peut tenter d'expliquer le succès obtenu par le mouv.ment 5 Etoiles si l'on considère en effet son histoire, depuis le net, jusqu'aux victoires aux élections politiques nationales et un certain succès aussi à celles européennes. Néanmoins ce succès repose, à notre sens, sur la construction d'un récit, d'un storytelling particulier. Ce storytelling est fondé sur trois piliers narratifs principaux : l'Internet comme outil pour une meilleure démocratie (continue, délibérative et participative); la déception vis-à-vis du système politique traditionnel ( anti-politique et anti-caste $»^{20}$ ) en luttant contre la corruption et la tentative d'une moralisation de la vie publique. Il est difficile de séparer ces facteurs en déterminant précisément celui qui a œuvré le plus efficacement; on préfère considérer que l'adhésion à ce mouvement politique s'est produit par un concours de circonstances socio-psychologiques, alimenté par une croyance absolue dans la neutralité des outils technologiques.

Mais on remarquera que ce pouvoir exceptionnel que l'on attribue à l'Internet est déjà la marque caractéristique de son propre storytelling. L'Internet est sans doute un outil, mais si ce qui définit un outil est sa finalité propre, il nous faudrait comprendre quelle est la finalité ultime (ou primaire) de l'Internet. S'il est très souvent décrit comme outil anarchique capable d'ébranler les vestiges du capitalisme, il est aussi le nouvel instrument que le néocapitalisme ${ }^{21}$ involontairement ou pas a produit, et pour une nouvelle forme de consommation, et pour une nouvelle forme de contestation amplifiée par l'anonymat et l'immatérialité des liens.

19 Ce programme de communication et surtout d'information citoyenne ne pouvait pas voir le jour sans les nouvelles techniques numériques de communication. L'enveloppement réticulaire $^{22}$ a saisi la société et toutes ses composantes et modes opératoires, et/ou organisationnels. Le dispositif technique d'internet a fini par réinterpréter tous les objets sociaux existants en impactant notre façon de vivre ensemble et a aussi fini par créer de nouvelles pratiques de la gestion de la chose publique.

20 Mais comment être sûr que tous les citoyens puissent vraiment faire remonter leurs opinions ? L'idée ou la bonne intention de faire activement participer les citoyens aux débats politiques est une réponse logique et juste, bien que marquée par un certain idéalisme optimiste sur la nature humaine et sur l'égalité des compétences que chaque citoyen serait censé posséder. Mais sommes-nous tous compétents sur tout? L'internet n'a-t-il pas alimenté abusivement l'idée que nous sommes des omniscients et que ce que nous ne connaissons pas, nous pouvons quand même parvenir à le connaître ? Autrement dit, en particulier pour le domaine politique, comme déjà le questionnait Platon, faut-il défendre une professionnalisation de la politique ou peut-on admettre, avec les 5 Etoiles, que chacun peut apporter véritablement sa compétence? Comment ne pas craindre que les technocrates de l'Internet s'accaparent la démocratie, au moyen d'un storytelling 
marqué par un idéal de liberté effrénée d'expression et par un faux mythe de neutralité de l'outil?

\section{Analyse de cas : les propositions législatives des 5 Etoiles sur la plateforme participative « Rousseau »}

21 Voyons, maintenant, si le storytelling fondateur des 5 Etoiles a su tenir sa parole quasi messianique d'une alternative possible à la politique traditionnelle, notamment en se concentrant sur l'appel originaire à la transparence de fonctionnement des structures associatives et participatives de tout mouvement politique, ainsi que sur l'effective et libre participation des netoyens (citoyens de l'Internet, ou plus correctement citoyens grâce à l'Internet).

22 Lorsqu'on navigue sur le site beppegrillo.it (1,9 millions de followers sur Facebook et 22,4 millions de followers sur Tweeter), blog fondateur et aujourd'hui encore la source idéologique du mouvement, on est étourdi par un flux d'informations et d'images dont on n'arrive pas à comprendre le critère organisationnel. On pourrait déjà se demander si ce manque d'organisation est intentionnel dans le but de brouiller l'esprit du lecteur et de l'empêcher effectivement de trouver un chemin pour se forger lui-même un esprit critique sur n'importe quelle question abordée. De plus, une multitude de pop-up s'ouvre (nous en avons compté sept), en montrant toutes sortes de publicité commerciale : on se serait attendu que ce blog fasse mieux écran à ce type de publicité d'autant plus que Beppe Grillo s'est toujours opposé aux multinationales capitalistes et gourmandes.

Deux volets ont attiré en particulier notre attention: l'archive et les sondages. Pour ce qui concerne le premier, l'archive est consultable à partir de deux menus: un qui sélectionne par mois et année de publication des messages, l'autre par thématique, à choisir parmi «écologie, économie, énergie, information, politique, santé/médecine, esclaves modernes, technologie/réseau, transports/viabilité, mur des pleurs, primaires des citoyens, mairies à 5 étoiles». Il serait intéressant de remarquer comment la thématique "politique » est en soi isolée : en cliquant on retrouve des posts absolument hétérogènes sur des élections, sur le cas des exploitations pétrolières de la mer Adriatique, sur les relations entre Renzi et Napolitano (ancien président de la République Italienne). Si on fait un autre essai en choisissant par exemple la thématique « information », on y retrouve la même hétérogénéité de contenu : un post sur la mort du fondateur Roberto Casaleggio, une attaque contre les industries pharmaceutiques, une alerte sur la possibilité d'une guerre civile en Grèce, des informations sur les exploitations pétrolières à nouveau, un article du «The Guardian » sur Virginia Raggi, Maire de Rome, en somme une pléthore d'informations, mais pas de messages sur ce que le mouvement 5 Etoiles pourrait vouloir en matière d'information.

Le second volet ouvre sur les sondages qui ont été proposés, unilatéralement, par le blog depuis 2011 jusqu'à seulement 2014. Là encore, l'hétérogénéité des propositions est évidente, car on y retrouve : « comment arrêter le pouvoir des banques ? ", «faut-il abolir l'argent comptant?», «quelle politique raconte le plus de mensonges?", «Boldrini (Président de la Chambre des Députés) rentre chez toi : que doit-elle faire? ", " sortons de l'Euro zone pour sauver les entreprises ", «quelles sont les causes du déclin économique italien? ", « comment abolir les financements privés aux partis ? », « le pire Président de la République», "que devrait faire la Chine pour le Tibet?», «sondage sur la loi 
électorale ", « la soutenabilité alimentaire ", « sondage sur l'abolition des sénateurs élus à vie ». On signalera aussi pour son caractère visiblement confus, mais éclairant sur le type d'information qui se propage du blog de Beppe Grillo, le sondage «Contre l'information des partis : comment résoudre le problème de surpopulation dans les prisons?». Nous avons cliqué sur plusieurs de ces sondages et les résultats ne sont plus consultables! Celui sur l'excès de pouvoir des banques n'est même plus cliquable. De plus, semble complètement désavoué le principe de consultation permanente des citoyens puisque, d'une part, les sondages proposés s'arrêtent à 2014 et, d'autre part, les résultats outre qu'ils ne sont plus disponibles, ne se concrétisent pas dans une action utile à la vie politique. En termes de tension narrative, on pourrait remarquer avec Raphaël Baroni que ces sondages tiennent ouvertes des "virtualités plus ou moins espérées ou redoutées... vers un dénouement à venir dont le caractère purement virtuel engendre un effet de suspense très efficace ${ }^{23}$ où " ce récit à suspens renvoie à une véritable éthique de l'action pour le lecteur-citoyen... le suspens constitue une réalité politique, il est tout simplement la marque d'un engagement du lecteur-citoyen dans une histoire encore ouverte. Les virtualités de l'histoire qui n'ont pas encore été actualisées, peuvent certes nous inquiéter, et elles génèrent du pathos et une attente de la suite de l'histoire... elles engagent notre responsabilité face à un avenir encore ouvert. »

Il est vrai que depuis que certains membres du mouvement ont été élus au Parlement, ils peuvent maintenant faire entendre leur voix dans un lieu institutionnel, et pour donner corps au principe qu'il a toujours défendu, le mouvement s'est équipé d'une plateforme participative, appelée "Rousseau». Cette plateforme, inaugurée en août 2017, est accessible à tous, inscrits ou pas au mouvement 5 Etoile, sauf que ceux qui ne sont pas inscrits, ne peuvent pas participer ni donner leur contribution au débat politique. Si vous naviguez sur cette plateforme, vous y retrouverez des propositions de lois, selon le type de Commission Parlementaire compétente. Parmi les propositions, reparties en Nationales et Européennes, figurent les suivantes: "gestion et cartographie des infections hospitalières", "adéquation des parcs d'enfants pour les handicapés", «élargissement de l'exploitation des mises sur écoutes ", "prévention du vol des vélos par le biais de l'introduction d'un dispositif électronique d'identification grâce à un code unique national », « Institution d'une commission parlementaire d'enquête sur les délits des banques, même étrangères et sur la surveillance de la Banque Centrale Italienne ", "modifications sur l'évaluation de l'impact sur le bien-être de la société des lois qui ont un coût de 500 millions d'euros pour l'Etat ». Si certaines propositions semblent tout à fait légitimes dans leur contenu, d'autres ne nous empêchent pas de sourire, en reproposant la question originaire : à quoi s'attèlent les députés italiens? quelles priorités ont-ils à l'esprit pour le bon gouvernement de la République?

Chaque proposition est illustrée par une vidéo et une courte synthèse qui devrait expliquer le contenu de loi souhaité. S'en suivent toutes les participations postées par les netoyens. Pour illustrer le mode de fonctionnement de participation démocratique souhaitée par les 5 Etoiles, nous avons choisi un sujet simple et compréhensible parmi les 316 propositions contenues sur la plateforme. Il s'agit de la proposition «TVA réduite pour les boissons alternatives au lait ", par exemple les boissons à base de soja, d'avoine, de riz dont raffolent certains véganes. Il faut préciser d'abord qu'en l'état actuel, la TVA pour le lait de vache est de $4 \%$, alors que pour les autres boissons elle est à hauteur de $22 \%$, comme il est clairement annoncé dans la synthèse de présentation. Les netoyens peuvent participer en proposant une objection, une modification, une suggestion ou une 
intégration : en langage juridique chaque type d'intervention est donc significativement différent. Si l'on se penche sur les participations des netoyens, nous pouvons constater que sur 49 participations, nous avons 4 objections, 30 suggestions, 14 intégrations et une seule modification. En voyant de plus près les contenus, on constate que les quatre typologies sont pas utilisées de façon correcte, ce qui met en doute les compétences des 5 étoiles en matière de discussions parlementaires. Au-delà de ces détails de style, concentrons-nous sur les arguments proposés: la seule modification (qui en réalité est une intégration) propose de rajouter le mot "biologique»; parmi les suggestions on retrouve des arguments qui vont du cas personnel (« je suis moi-même allergique au lactose, donc il faut baisser la $\mathrm{TVA}^{24} »$ ), à l'extension à d'autres questions certes importantes, mais pas forcément pertinentes sur la baisse de TVA pour les boissons végétales : « il faut baisser la TVA, mais aussi donner des aides aux parents pour renforcer la natalité »; « il faut faire attention à l'exploitation des élevages »; «il faut penser aux véganes »; «il faudrait, en effet, enlever aussi la TVA pour le gaz et l'énergie électrique »; " pour les gens qui sont allergiques au lactose ces boissons sont nécessaires et il faudrait aussi prendre en compte une baisse de TVA pour ceux qui souffrent de rétinopathie "; « il faut baisser la TVA en question, mais aussi imposer une nouvelle taxe sur la soutenabilité éthique et environnementale ». 18 participants se limitent tout simplement à des suggestions/ modifications/intégrations dont le contenu est «Bien, OK, je suis d'accord, bravo!». Parmi les objections, on signalera la suivante: "les boissons végétales sont un phénomène de mode et on ne devrait pas trop s'y investir ». La seule personne qui apporte des éléments scientifiques est un ingénieur techno-agro-alimentaire qui précise les vices et vertus du lait de vache et de ces boissons végétales. Deux personnes avouent ne pas connaitre précisément le taux prévu de TVA ou ignorent que la Commission Européenne a déjà interdit le mot « lait " pour ces boissons.

Ces exemples en disent, en effet, beaucoup sur les compétences des participants, non seulement en termes de technique législative (là où il $\mathrm{y}$ aurait bien de vices de forme), mais aussi en matière de boissons végétales. De plus, ce qui a attiré notre attention est la sorte d'euphorie de toute puissance qui souffle dans ces propositions citoyennes, comme si poster un commentaire à une proposition législative était suffisant pour qu'elle soit prise en compte. Autrement dit, nous voulons attirer l'attention sur la différence capitale qui existe en science de la communication entre «exprimer son avis » et " communiquer ». C'est seulement dans le second cas que la communication est efficace parce que le destinataire a reçu un message et renvoie à l'émetteur un message de réponse pertinente. Dans le cas de l'expression, en revanche, chacun peut certes donner son avis, mais pour qu'elle soit efficace, il n'est nul besoin de penser au destinataire! Un des périls de la communication au sens large sur les réseaux sociaux est en effet de toujours confondre ces deux termes: chacun pense être libre de s'exprimer, mais le paradoxe est que personne n'a envie non plus de lire, d'écouter ou d'intégrer les réponses d'autrui.

\section{Conclusions}

Le mouvement 5 Etoiles a su construire de par son histoire un storytelling apparemment révolutionnaire, basé sur l'indignation, la peur, et en se proposant comme un mouvement de libération de tous les vices du capitalisme ou du néocapitalisme. Les nouvelles 
technologies y ont joué un rôle capital, accompagnées elles-mêmes par un storytelling tout aussi innovant, libertaire et démocratique.

La suite des événements a néanmoins montré que le storytelling initial des Grillini n'a pas été en mesure de tenir ses engagements originaires, en voyant se multiplier les accusations d'autoritarisme du leadership, d'opacité dans le mode de fonctionnement (de la procédure d'élection primaire au fonctionnement des plateformes du Mouvement, blog, site et "Rousseau » compris), éloignement constant des principes fondateurs et rapprochement tout aussi constant aux logiques de communication, d'élection et de fonctionnement des partis traditionnels. Et pourtant l'idée semblait bonne : redonner la parole au peuple!

\section{BIBLIOGRAPHY}

Biorcio R., Natale P., Politica a 5 stelle. Idee, storia e strategia del movimento di Grillo, Milan, Feltrinelli, 2013.

Cazeaux G., Odyssée 2.0. La démocratie dans la civilisation numérique, Paris, Armand Colin, 2014.

Corbetta P., Gualmini E., Il partito di Grillo, Bologne, Il Mulino, 2013.

Crouzet Th., Le cinquième pouvoir. Comment internet bouleverse la politique, Paris, Bourin Editeur, 2007.

Derville G., Le pouvoir des médias, Grenoble, Presses universitaires de Grenoble, 2013.

Ippolita (collectif), Internet. L'illusion démocratique, Paris, Editions de la différence, 2016.

Le Coz P., Le gouvernement des émotions et l'art de déjouer les manipulations, Albin Michel, 2014.

Marti M., Pelissier N. (dirs.), Tension narrative et storytelling. En attendant la fin, l'Harmattan, 2013.

Mazzoleni G., La comunicazione politica, Bologne, Il Mulino, 2004.

Musso P., Réseaux et société, Paris, PUF, 2003.

Orazi F., Socci M., Il popolo di Beppe Grillo, Ancona, Cattedrale, seconde édition, 2008.

Orazi F., Socci M., Il grillismo. Tra democrazia elettronica e movimento personale, Rome, Carocci Editore, 2014

Proulx S., « Pratiques d'internet et numérisation des sociétés » in Lajoie J., Guichard E., Odyssée Internet. Enjeux sociaux, Québec, Presses de l'Université du Québec, 2002.

Richard S., Numériques, Paris, Bernard Grasset, 2014.

Rodotà S., La démocratie numérique. De nouveaux concepts et expériences politiques, trad. Fr. Guyot J., Paris, Ed. Apogée, 1999.

Salmon Ch., Storytelling, la machine à fabriquer les histoires et à formater les esprits, La Découverte, Paris, 2007 
Santoro G., Un Grillo qualunque. Il Movimento 5 Stelle e il populismo digitale nella crisi dei partiti italiani, Rome, Lit Edizioni, 2001.

\section{NOTES}

1. B. Lamizet, Le langage politique. Discours, images, pratiques, Ellipses, 2011 ; L. Cedroni, T. Dell'Era, Il linguaggio politico, Rome, Carocci, 2002.

2. G. Orwell, 1984, Gallimard, 1972.

3. Christian Salmon, Storytelling, la machine à fabriquer les histoires et à formater les esprits, La Découverte, Paris, 2007, p. 12.

4. Ibidem, pp. 16-17.

5. Ibidem p. 42.

6. Pierre Le Coz, Le gouvernement des émotions et l'art de déjouer les manipulations, Albin Michel, 2014, p. 85.

7. Idem, p. 87.

8. Cette reconstruction s'inspire d'une analyse précédemment présentée au XIXème congrès de la Société Française des Sciences de l'Information et de la Communication, qui a eu lieu à Toulon en 2014.

9. Gianpietro Mazzoleni, La comunicazione politica, Bologne, Il Mulino, 2004, p. 23.

10. Biorcio, Natale, op. cit., 2013, p. 21.

11. Piergiorgio Corbetta, Elisabetta Gualmini, Il partito di Grillo, Bologne, Il Mulino, 2013, p. 43.

12. Idem, p. 44.

13. Biorcio, Natale, op. cit., 2013, p. 87.

14. Francesco Orazi, Marco Socci, Il grillismo. Tra democrazia elettronica e movimento personale, Rome, Carocci Editore, 2014, p. 84.

15. Biorcio, Natale, op. cit., 2013, p. 91.

16. Corbetta, Gualmini, op. cit., pp. 212-213.

17. Francesco Orazi, Marco Socci, Il grillismo .op. cit., en particulier pp. 71-80.

18. Rodotà Stefano, La démocratie numérique. De nouveaux concepts et expériences politiques, trad. Fr. Guyot J., Paris, Ed. Apogée, 1999, p. 94.

19. S. Proulx, "Pratiques d'internet et numérisation des sociétés ", in J. Lajoie, E. Guichard, Odyssée Internet. Enjeux sociaux, Québec, Presses de l'Université du Québec, 2002, p. 33.

20. Biorcio, Natale, op. cit., 2013, pp. 140-151.

21. CF. Ippolita (collectif), Internet. L'illusion démocratique, Paris, Editions de la différence, 2016.

22. Musso Pierre, Réseaux et société, Paris, PUF, 2003.

23. Raphaël Baroni, « La tension narrative à travers les genres : enjeux éthiques et esthétiques du suspense », pp. 17-35 in Marc Marti, Nicolas Pelissier (dirs.), Tension narrative et storytelling. En attendant la fin, l'Harmattan, 2013.

24. Nous avons librement traduit de l'italien et résumé les contenus des posts des participants. 


\section{ABSTRACTS}

Depuis une décennie, le Mouvement 5 Etoiles fondé par l'ex-comédien Beppe Grillo a obtenu un large succès de public, en devenant une des principales forces politiques parlementaires en Italie. Ce succès est le fruit d'une précise technique de storytelling, adressée aux désabusés de la politique italienne (les indignés) et fondée sur l'indignation, la lutte à la corruption politique et économique, l'espoir dans une nouvelle moralisation de la vie publique ainsi que la transparence de gouvernance. En parcourant les principales étapes historiques du mouvement, l'auteur analyse le storytelling utilisé par Beppe Brillo. Dans la seconde partie de l'article, l'auteur analyse une proposition de loi présentée sur la plateforme participative "Rousseau » pour montrer comment, en fait, le storytelling initial ait ouvert des doutes sur la faisabilité et la réelle application des principes fondateurs du mouvement.

Da circa un decennio il movimento 5 Stelle fondato dall'ex comico Beppe Grillo ha riscosso un notevole successo, diventando una delle principali forze politiche parlamentari italiane. Tale successo é il frutto d'una precisa tecnica di storytelling che si fonda sull'indignazione, la lotta alla corruzione politica ed economica, la speranza di una nuova moralizzazione, e, soprattutto, la trasparenza di governo. Attraverso la ricostruzione delle principali fasi storiche del moviemento l'autore intende mostrare il tipo di storytelling utilizzato da Beppe Grillo. Nella seconda parte dell'articolo, viene analizzata una proposta di legge presente sulla piattaforma « Rousseau » per mostrare come, in realtà, lo storytelling iniziale abbia invece aperto dubbi effettivi sulla reale e possibile applicazione dei principi tanto propugnati.

\section{INDEX}

Geographical index: Italie

Chronological index: XXIème siècle

Mots-clés: communication politique, e-démocratie, réseaux sociaux participatifs, Mouvement 5 Etoiles

\section{AUTHOR}

\section{VALENTINA TIRLONI}

Après des études de philosophie et de droit et une thèse en Philosophie des Sciences sociales, Valentina Tirloni est maître de conférences en Sciences de la communication à l'Université Nice Sophia Antipolis et membre du CRHI. Ses recherches portent sur l'impact de la technique et de la technologie sur l'individu (transhumanisme) et sur la société (e-démocratie). 